\title{
Soft Target Weight: Theory and Simulation of a Novel Haemodialysis Protocol Which Reduces Excessive Ultrafiltration
}

\author{
Damien Ashby Richard Corbett Neill Duncan \\ West London Renal and Transplant Centre, Imperial College Healthcare NHS Trust, Hammersmith Hospital, London, UK
}

\section{Keywords}

Haemodialysis · Ultrafiltration · Dry weight · Intra-dialytic hypotension - Dialytic symptoms

\begin{abstract}
Introduction: Excessive ultrafiltration is associated with intra-dialytic symptoms, loss of residual function, and mortality in haemodialysis patients. A major contributor to excessive ultrafiltration is within-individual variation in pre-dialysis weight and the concept of achieving a fixed target weight by the end of each dialysis session. Haemodialysis protocols which allow variable post-dialysis weight have not been proposed. Methods: Weight variation was observed in haemodialysis patients and healthy controls to estimate the proportion of pre-dialysis weight variation which could be considered natural variation. These estimates were used to derive a novel protocol for setting ultrafiltration, which was evaluated by mathematical modelling. Results: Amongst 20 haemodialysis patients, mean (SD) pre-dialysis weight was 102.74 (0.94)\% of target weight after a 2-day gap and 103.50 (0.94)\% after a 3-day gap. Amongst 10 healthy individuals, mean (SD) daily weight was $100.0(0.71) \%$ of average weight. A 4-component model of pre-dialysis weight was derived using these estimates, in which the best estimate of pre-dialysis excess fluid is the midpoint of excess weight and average fluid gain, and used to propose a novel protocol for ultrafiltration setting. In simulations, the novel protocol reduced
\end{abstract}

karger@karger.com www.karger.com/nef

Karger $\stackrel{\text { ' }}{5}$

GOPEN ACCESS
(C) 2021 The Author(s)

Published by S. Karger AG, Basel

This is an Open Access article licensed under the Creative Commons Attribution-NonCommercial-4.0 International License (CC BY-NC) (http://www.karger.com/Services/OpenAccessLicense), applicable to the online version of the article only. Usage and distribution for commercial purposes requires written permission. ultrafiltration variation by more than half (standard deviation 0.6 vs. $1.3 \%$ of target weight, $p<0.001$ ), without increasing the variation in post-dialysis fluid excess. Excessive ultrafiltration rates (over $13 \mathrm{~mL} / \mathrm{h} / \mathrm{kg}$ ) were far less frequent using this protocol ( $2.6 \%$ vs. $7.5 \%$ of sessions, $p=0.001)$. Conclusion: Considering natural weight variation allows the development of a novel protocol for ultrafiltration in which target weight does not have to be achieved precisely: it is therefore a soft target. This protocol, which is predicted to substantially reduce excessive ultrafiltration variation, is a zero-cost intervention with the potential to improve symptoms and clinical outcome for haemodialysis patients.

C 2021 The Author(s).

Published by S. Karger AG, Basel

\section{Introduction}

Ultrafiltration rate and volume are increasingly recognized as modifiable treatment parameters of great clinical importance. High rates of ultrafiltration in particular are associated with symptomatic intra-dialytic hypotension [1], secondary pathologies such as cardiac injury or loss of residual function [2], and clinical outcomes including mortality $[3,4]$. Much haemodialysis research effort is focussed on methods to reduce harms arising from excessive ultrafiltration, including intra-dialytic approaches such as ultrafiltration profiling $[5,6]$, and accurate objec- 
tive measures of target weight such as bioimpedance [7]. In addition, several treatments are advocated to reduce ultrafiltration rate, including diuretics, lengthened session duration, and increased dialysis frequency [8].

Ultrafiltration volume and rate are not constant for individuals, however, but vary according to pre-dialysis weight, with ultrafiltration almost universally set equal to excess weight (the difference between pre-dialysis weight and target weight). Thus, high ultrafiltration volume and rate are partly a consequence of this variation, with most patients experiencing the effects of rapid ultrafiltration during some sessions, and many commenting "I always feel terrible when they take off more than..." Indeed patients sometimes wonder why ultrafiltration is not the same fixed volume each dialysis session - after all, the total monthly ultrafiltration would be the same.

Intuitively, variation in ultrafiltration seems necessary because fluid intake varies, evidenced by variation in predialysis weight. But patients also question this ("I'm 3 kilos up, but I really didn't drink that much"), and since weight varies in healthy individuals [9], at least a proportion of the variation in pre-dialysis weight may be natural, rather than pathological. In this study, we sought to estimate dry weight variation in dialysis patients by comparison with weight variation in healthy controls, to derive a novel protocol for ultrafiltration which treats dry (normally hydrated) weight as a variable rather than fixed quantity, and to simulate the effect of this novel protocol on haemodialysis parameters such as ultrafiltration volume and rate.

\section{Materials and Methods}

Dialysis records were analysed for a random selection of haemodialysis patients from a single mixed-ethnicity urban dialysis satellite unit over a 1-year period. Weight variation in healthy controls was analysed from data provided by hospital staff involved in the care of dialysis patients, who were asked to weigh themselves daily, at the same time of day, with an empty bladder, over a period of at least 2 weeks. The study was conducted according to the principles of the Declaration of Helsinki, with the protocol being reviewed and approved by the institutional governance committee.

Although weight variation in healthy individuals includes fluid components, it is reasonable to think that this variation occurs without pathological fluid disorder, consisting of variable fluid within the gastrointestinal lumen (where it is unavailable for removal during dialysis) as well as variation in dry components of weight (such as total body carbon). Thus, the variance of weight in healthy individuals can be regarded as an estimate of the variance in dry (normally hydrated) weight in dialysis patients.

Since pre-dialysis weight variation contains both dry and fluid components, pre-dialysis weight can be illustrated by a 4-compo-

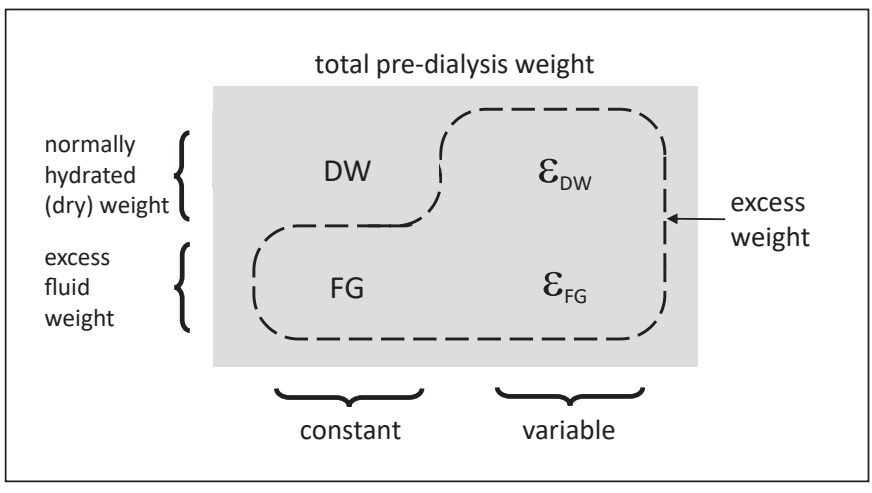

Fig. 1. Model of pre-dialysis weight. Four-component $(2 \times 2)$ model of pre-dialysis weight, separated into rows (normally hydrated vs. excess fluid components) and columns (constant vs. variable components). DW, dry weight; FG, (average) fluid gain; $\varepsilon_{\mathrm{DW}}$ and $\varepsilon_{\mathrm{FG}}$, variable ("error") terms which may be positive or negative, with mean zero. Excess weight is not the same as excess fluid, since it also includes the variable component of normally hydrated (dry) weight $\left(\varepsilon_{\mathrm{DW}}\right)$.

nent $(2 \times 2)$ model (Fig. 1). With this model, it can be seen that not all excess weight is fluid excess, so that simple use of excess weight (EW) as the ultrafiltration target may lead to either excessive or insufficient fluid removal. The actual fluid excess cannot conveniently be measured each dialysis session.

However, although fluid excess cannot be measured precisely, it can be estimated. In probability theory, for 2 variables $A$ and $B$ with mean zero, if the sum $A+B$ is known, but the individual components $A$ and $B$ are unknown, then the expected value of $B$ is given by

$$
E(B \mid A+B)=(A+B) \times \mathrm{VB} /(\mathrm{VA}+\mathrm{VB}),
$$

where $E(B \mid A+B)=$ expected value of $B$, given the value of $A+B$, $\mathrm{VA}=$ variance of $A$, and $\mathrm{VB}=$ variance of $B$.

This is a type of signal extraction problem since the pure signal $(B)$ is being extracted from the signal plus noise $(A+B)$. Applying this to our 4-component model of haemodialysis (Fig. 1), if $\varepsilon_{\mathrm{DW}}$ and $\varepsilon_{\mathrm{FG}}$ are the variable components of dry weight and fluid gain, then the expected excess fluid weight to be removed is given by

$$
\begin{aligned}
& E\left(\mathrm{FG}+\varepsilon_{\mathrm{FG}} \mid \mathrm{FG}+\varepsilon_{\mathrm{FG}}+\varepsilon_{\mathrm{DW}}\right)= \\
& \mathrm{FG}+\left(\varepsilon_{\mathrm{FG}}+\varepsilon_{\mathrm{DW}}\right) \times \mathrm{V} \varepsilon_{\mathrm{FG}} /\left(\mathrm{V} \varepsilon_{\mathrm{DW}}+\mathrm{V} \varepsilon_{\mathrm{FG}}\right),
\end{aligned}
$$

where $\mathrm{FG}$ and $\varepsilon_{\mathrm{FG}}=$ fixed and variable components of fluid gain, and $\mathrm{DW}$ and $\varepsilon_{\mathrm{DW}}=$ fixed and variable components of dry weight.

Since $\varepsilon_{\mathrm{DW}}$ and $\varepsilon_{\mathrm{FG}}$ (the variable components of dry weight and fluid gain) were observed to have approximately equal variance, the expected excess fluid weight is given by

$$
E\left(\mathrm{FG}+\varepsilon_{\mathrm{FG}}\right)=\mathrm{FG}+\left(\varepsilon_{\mathrm{FG}}+\varepsilon_{\mathrm{DW}}\right) / 2 .
$$

Since FG $+\varepsilon_{\mathrm{FG}}+\varepsilon_{\mathrm{DW}}$ is excess weight (EW), this simplifies to

$E\left(\mathrm{FG}+\varepsilon_{\mathrm{FG}}\right)=(\mathrm{FG}+\mathrm{EW}) / 2$. 
hard target weight protocol

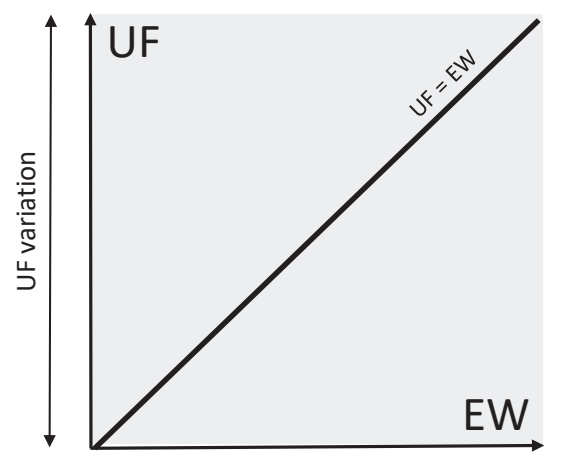

soft target weight protocol

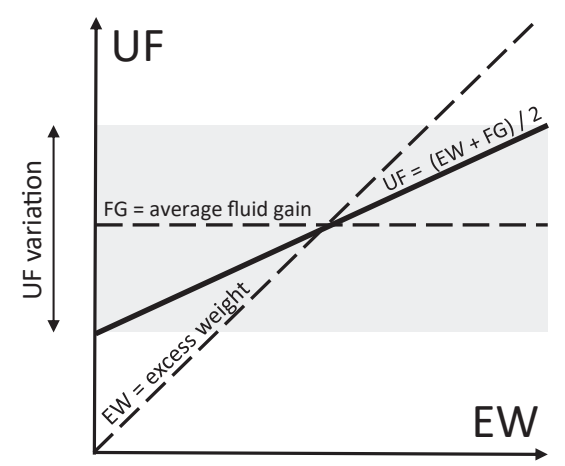

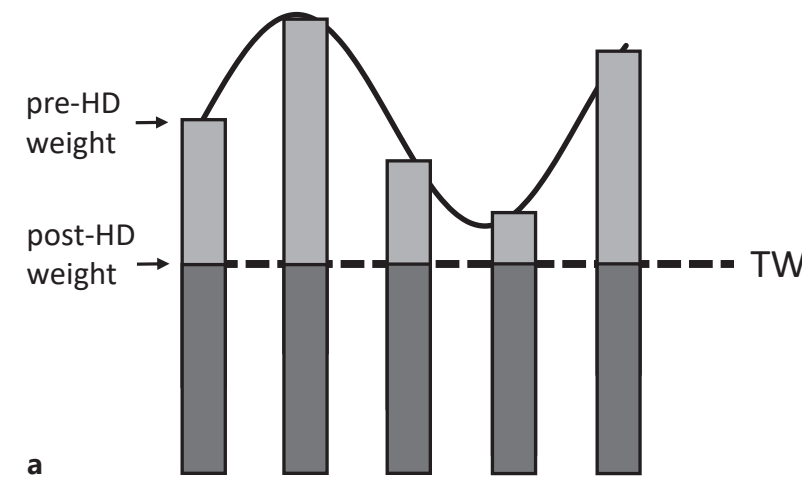

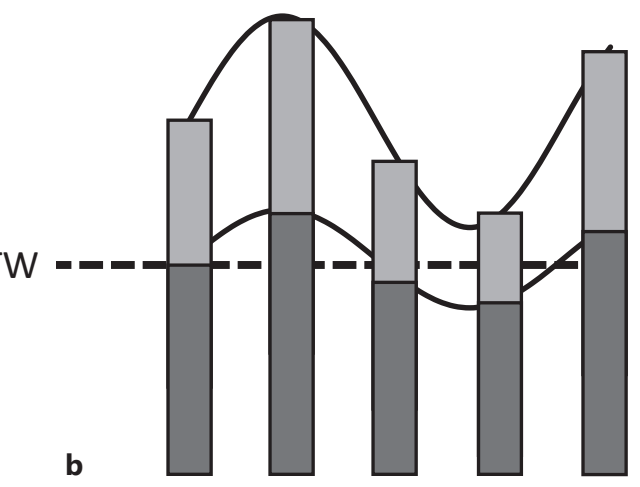

Fig. 2. Ultrafiltration protocols. Upper panels: relationship between EW and UF. Lower panels: effect on dialysis parameters over 5 sessions. a Hard TW protocol, in which dry weight (dark grey) is assumed to be the same each day, all excess weight is assumed to be fluid, and ultrafiltration (light grey) is set equal to excess weight, so that target weight is achieved each session. b Soft target weight protocol, in which dry weight is accepted to vary, and ultrafiltration is therefore set to the midpoint of excess weight and average FG, so that target weight is approached but not achieved each session. EW, excess weight; UF, ultrafiltration; TW, target weight; FG, fluid gain.

A novel haemodialysis protocol is therefore proposed, in which rather than setting ultrafiltration to excess weight, ultrafiltration is instead set to the midpoint of excess weight and average fluid gain:

$$
\mathrm{UF}=(\mathrm{EW}+\mathrm{FG}) / 2 .
$$

For example, suppose a patient with target weight $50 \mathrm{~kg}$ and average intra-dialytic weight gain $1.5 \mathrm{~kg}$ arrives for dialysis weighing $2.5 \mathrm{~kg}$ above target. Ultrafiltration according to the 2 protocols would be as follows:

Traditional protocol: $2.5 \mathrm{~L}$ (= excess weight);

Novel protocol: $2.0 \mathrm{~L}$ (= midpoint of excess weight and average fluid gain).

Since target weight does not have to be achieved with this protocol, it is a soft target - this is therefore the "soft target weight" protocol. However, the standard protocol, in which ultrafiltration is set equal to excess weight, is the "hard target weight" protocol. The protocols are graphically illustrated in Figure 2.
Modelling was performed using the 4-component model described, with fixed and random components of both dry and fluid weights and with excess fluid remaining after dialysis carried over to the fluid weight at the start of the next session. Weight changes during thrice-weekly haemodialysis were simulated over 6-month periods, with fluid removal parameters assessed over a number of simulations. Simulation parameters informed by observations included dry weight (DW $\left.+\varepsilon_{\mathrm{DW}}\right)$, which was set stable over 6 months with standard deviation $0.71 \%$, and fluid excess $\left(\mathrm{FG}+\varepsilon_{\mathrm{FG}}\right)$, which was given a mean varying between individuals (given by a normal distribution with mean $2.7 \%$, SD $1.0 \%$ for 2-day gaps, 1.3 -fold higher for 3-day gaps) and intra individual SD $0.62 \%$. Microsoft Excel v16 was used for modelling and statistical analysis, with the $F$ test used to compare standard deviations. 


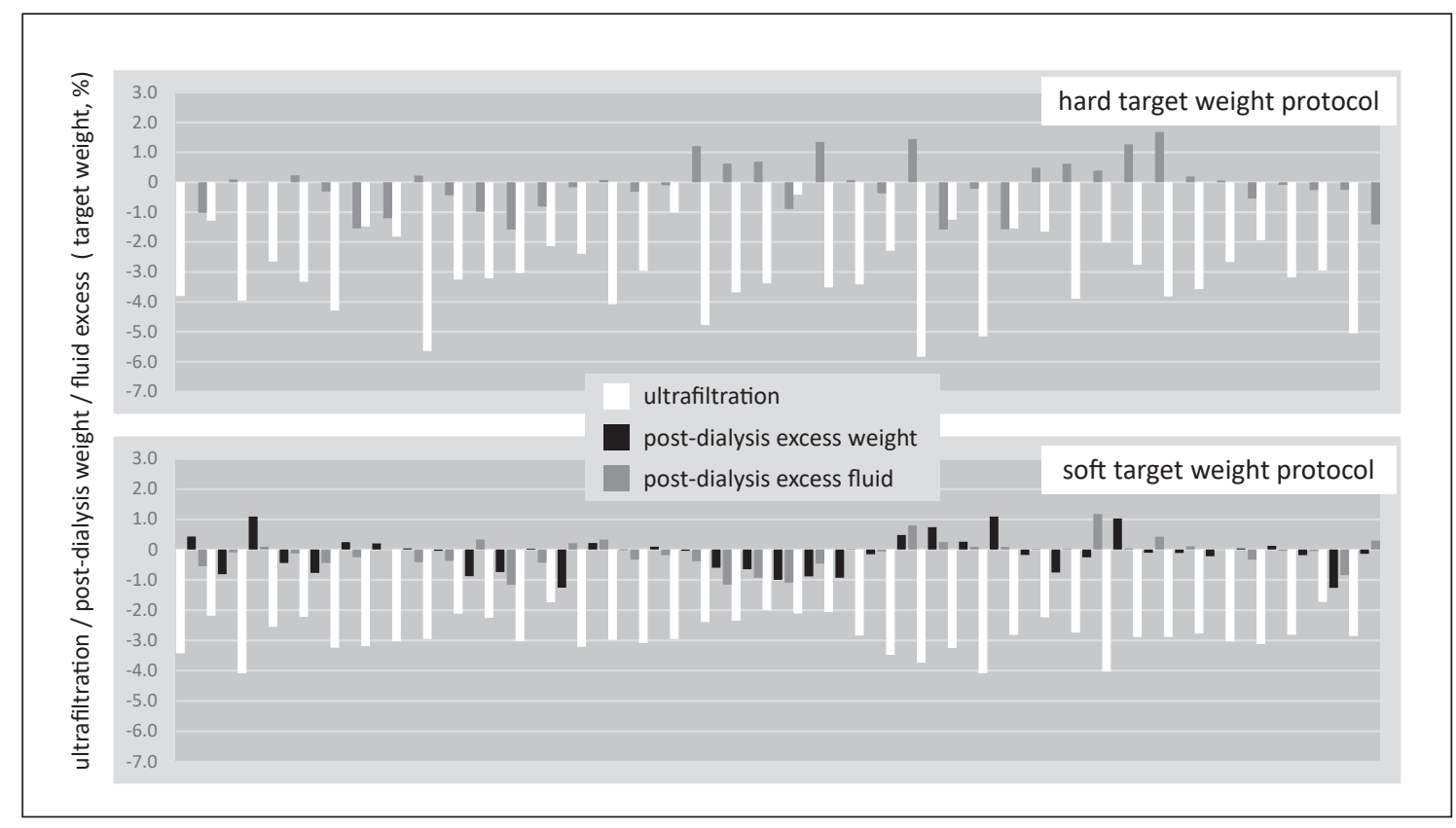

Fig. 3. Haemodialysis simulation. Representative simulation of thrice-weekly haemodialysis over 3 months using hard target weight (upper panel) and soft target weight (lower panel) protocols, showing ultrafiltration (white), post-dialysis excess weight (black), and excess fluid (grey). The soft target weight protocol introduces a variable post-dialysis excess weight which is absent with the (traditional) hard target weight protocol, but it also greatly reduces ultrafiltration variation. Post-dialysis excess fluid, according to the 4-compartment model proposed, is similar with either protocol.

\section{Results}

\section{Weight Variation in Haemodialysis Patients and} Healthy Controls

Amongst 20 haemodialysis patients (aged $59 \pm 14,14$ male), mean pre-dialysis weight was $102.74 \%$ of target weight after a 2 -day gap and $103.50 \%$ after a 3 -day gap, with within-individual standard deviation $0.94 \%$ for both (variance $0.88 \%$ ). Mean (SD) post-dialysis weight was $100.00(0.56) \%$ (variance $0.31 \%$ ).

Amongst 10 healthy individuals (aged $38 \pm 11,3$ male), within-individual standard deviation of daily weight was $0.71 \%$ (variance $0.50 \%$ ). Daily weight variance in healthy individuals was therefore just over half (57\%) of the variance in patients' pre-dialysis weight, and much greater than the variance in patients' post-dialysis weight. Therefore, the total within-individual variance in pre-dialysis weight is split, approximately equally, between dry weight variance and fluid gain variance.

\section{Modelling Haemodialysis with Both Protocols}

The novel protocol was evaluated by mathematical modelling (an example simulation is illustrated in Fig. 3).
Comparing protocols in 100 patient simulations, each over 6 months, mean ultrafiltration was identical at 3.0\% in both groups, but compared to the hard target weight protocol, the standard deviation of ultrafiltration using the soft target weight protocol was reduced by more than half ( 0.6 vs. $1.3 \%, p<0.001$, Table 1$)$. As expected, a postdialysis weight variation of $\pm 0.6 \%$ was introduced by the novel protocol, but there was no increase in the variation of post-dialysis fluid excess, which was in fact marginally reduced ( \pm 0.6 vs. $\pm 0.7 \%, p<0.001$ ). Assuming a 4 -h duration for modelled sessions, excessive ultrafiltration rates (over $13 \mathrm{~mL} / \mathrm{h} / \mathrm{kg}$ ) were far less frequent using the soft target weight protocol ( $2.6 \%$ vs. $7.5 \%$ of sessions, $p=$ $0.001)$.

To illustrate the clinical impact more clearly, the effect of the 2 protocols on ultrafiltration distribution is compared for an example patient in Figure 4. For an $87-\mathrm{kg}$ patient treated with 4 -h sessions with average fluid gain 3 $\mathrm{kg}(3.5 \%)$, excessive ultrafiltration rates (over $13 \mathrm{~mL} / \mathrm{h} /$ $\mathrm{kg}$ ) were seen with the standard protocol in $9.7 \%$ of sessions, and with the soft target weight protocol in $0.2 \%$ of sessions. 
Fig. 4. Protocol comparison. UF distribution; for example, a patient weighing $87 \mathrm{~kg}$ with average fluid gain $3 \mathrm{~kg}(3.5 \%)$, treated with 4 -h sessions, showing the proportion of haemodialysis sessions with UF $<10,10-$ 13 , and $>13 \mathrm{~mL} / \mathrm{kg} / \mathrm{h}$ with each protocol ( white = hard target weight; grey = soft target weight). UF, ultrafiltration.

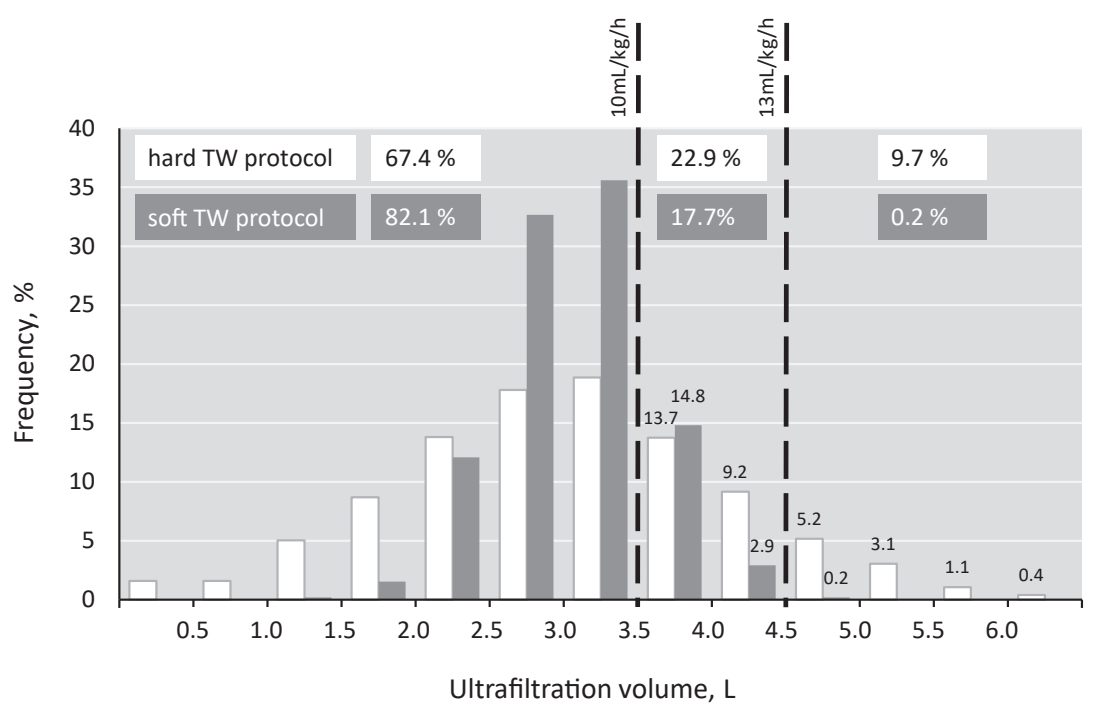

Table 1. Effect of traditional (hard target weight) and novel (soft target weight) protocols on haemodialysis parameters estimated by modelling ( $N=100$ simulations for each protocol)

\begin{tabular}{|c|c|c|c|c|c|}
\hline & \multicolumn{2}{|c|}{ Hard target weight protocol } & \multicolumn{2}{|c|}{ Soft target weight protocol } & \multirow[t]{2}{*}{$p$ value } \\
\hline & mean (SD) & 5th/95th pc & mean (SD) & 5th/95th pc & \\
\hline Ultrafiltration & $-3.0(1.3)$ & $-5.1 /-0.9$ & $-3.0(0.6)$ & $-3.9 / 2.1$ & $<0.001^{*}$ \\
\hline Pre-dialysis weight excess & $3.0(1.3)$ & $0.9 / 5.1$ & $3.0(1.1)$ & $1.2 / 4.8$ & $0.001^{*}$ \\
\hline Post-dialysis weight excess & $0.0(0.0)$ & $0.0 / 0.0$ & $0.0(0.6)$ & $-0.9 / 0.9$ & $<0.001^{*}$ \\
\hline Pre-dialysis fluid excess & $3.0(1.0)$ & $1.3 / 4.7$ & $3.0(0.9)$ & $1.5 / 4.5$ & $<0.001^{*}$ \\
\hline Post-dialysis fluid excess & $0.0(0.7)$ & $-1.2 / 1.2$ & $0.0(0.6)$ & $-0.9 / 0.9$ & $<0.001^{*}$ \\
\hline Percentage of sessions with rapid UF (>13 mL/h/kg) & $7.5(10.8)$ & $0.0 / 30.8$ & $2.6(8.9)$ & $0.0 / 15.0$ & $0.001^{+}$ \\
\hline
\end{tabular}

Volumes and weights are given as $\%$ of target weight. ${ }^{*} F$ test for difference in standard deviation. ${ }^{+}$Student's $t$ test for difference in means.

\section{Discussion}

In this study, appreciation of weight variation in healthy individuals led to an improved understanding of dialysis weight and a logical protocol for ultrafiltration setting. In simulated haemodialysis, the novel protocol substantially reduced variation in ultrafiltration without increasing variation in post-dialysis fluid excess and substantially reduced excessive ultrafiltration rates.

Whilst it has been known for decades that chronic fluid overload is harmful, it is now increasingly recognized that excessive ultrafiltration and high ultrafiltration rates in particular are also associated with poor outcome [10]. For example, in a retrospective analysis of data from the
HEMO study, Flythe et al. [11] reported substantially increased mortality when ultrafiltration rates exceeded 13 $\mathrm{mL} / \mathrm{h} / \mathrm{kg}$ (HR 1.59, 95\% CI: 1.29-1.96). Haemodialysis clinicians must therefore attempt to steer between the competing goals of achieving control of fluid overload, along with avoiding excessively rapid or severe ultrafiltration.

In attempting to address this problem, current research has focussed on 3 main approaches. First, profiled (nonlinear) ultrafiltration rates have been explored, such as those in which more fluid is removed at the start of dialysis when volume overload is the greatest, with reducing rate as the session progresses [12]. Second, reduced ultrafiltration rates have been achieved by increasing di- 
alysis frequency, lengthening sessions, or reducing interdialytic fluid gains [13]. Third, more accurate target weight setting has been sought, mostly by using technology to provide objective dry weight estimation. Bioimpedance is one of the most studied methods, and studies are underway which aim to demonstrate improved residual function by using this method to limit ultrafiltration $[14,15]$. But the wisdom of setting ultrafiltration volume equal to the total excess weight pre-dialysis (aiming for a fixed post-dialysis weight) has not been questioned before.

In this study, dry weight variability is estimated using data from healthy controls. Rather than seeking to measure it more accurately, dry weight is accepted as varying and not accurately identifiable, and ultrafiltration is set to the statistically expected value of excess fluid weight (the midpoint of excess weight and average fluid gain). This study therefore suggests a shift in the current paradigm of fluid removal by haemodialysis, suggesting that excess weight is not the same as excess fluid, and that a specific post-dialysis weight does not need to be achieved. Target weight is therefore a soft target, rather than a hard one, and this is therefore the "soft target weight" protocol.

This study generates a novel hypothesis, but its key limitation is its theoretical nature, relying as it does on reason and modelling. Whilst appearing promising in silico, the concepts of this study would require short- and long-term testing in the clinical setting before drawing conclusions. However, the hypothesis that variation in ultrafiltration can be reduced by half deserves attention, and the simplicity of the intervention is noteworthy: the potential to reduce symptoms and improve clinical outcome is often claimed for expensive new drugs or technologies, but the novel protocol described in this study is without cost and widely applicable.

Studies testing the clinical effects of this novel protocol are required, and potential detrimental effects, such as poorer long-term fluid control, would require careful exclusion. It is possible, for example, that use of the novel protocol might alter the detection of fluid overload and the performance of the current methods for determining dry weight. Individual factors such as blood pressure, salt intake, and plasma sodium may also interact with the protocol. Future clinical studies would therefore need to be carefully designed to assess safety and performance of the protocol in different patient circumstances. In addition, further refinements to the protocol might be favourable, such as a day-of-the-week adjustment, and there may be alternative protocol approaches which similarly view dry weight as variable and could reduce ultrafiltration variation. Although the estimate of daily weight variation in healthy controls is consistent with other studies [16], as an estimate of "normal" weight variation in dialysis patients, this may be inaccurate. However, it provides an estimate sufficient for hypothesis generation, leading to the proposed novel protocol. The key strength of this study is not so much the specific protocol, as the concept that dry weight is variable and that fixed target weight achievement may not be necessary.

In conclusion, observation of healthy individuals demonstrates that dry weight is not constant, and that up to half of the variation in pre-dialysis weight may be due to dry weight variation rather than variation in fluid gain. Using this concept, the best estimate of excess fluid is the midpoint of excess weight and average fluid gain, from which a simple novel ultrafiltration protocol (soft target weight) is proposed, in which target weight is approached but not precisely achieved. Simulations suggest that this protocol would substantially reduce variation in ultrafiltration and excessive ultrafiltration rates, representing a zero-cost intervention with the potential to improve symptoms and clinical outcome for haemodialysis patients.

\section{Acknowledgment}

No other individual or institution made a substantive contribution to this work.

\section{Statement of Ethics}

The study was conducted according to the principles of the Declaration of Helsinki. As a simulation study informed by retrospective data, the protocol was reviewed and approved by the institutional governance committee, with ethics committee approval not required.

\section{Conflict of Interest Statement}

D.A. has received a speaker honorarium from Fibrogen unconnected with this work. The authors have no other conflicts of interest to declare.

\section{Funding Sources}

No funding was received for this study. 


\section{Author Contributions}

D.A. conceived the study which was improved by discussions with R.C. and N.D. D.A. collected the data, performed the analysis and modelling, and wrote the initial manuscript, which was revised and agreed by R.C. and N.D.

\section{Data Availability Statement}

Data will be made available on request to the corresponding author.

\section{References}

1 Leunissen KM, Kooman JP, van Kuijk W, van der Sande F, Luik AJ, van Hooff JP. Preventing haemodynamic instability in patients at risk for intra-dialytic hypotension. Nephrol Dial Transplant. 1996;11(Suppl 2):11-5.

2 Mavrakanas TA, Sniderman AD, Barré PE, Vasilevsky M, Alam A. High ultrafiltration rates increase troponin levels in stable hemodialysis patients. Am J Nephrol. 2016;43(3): 173-8.

3 Chazot C, Vo-Van C, Lorriaux C, Deleaval P, Mayor B, Hurot JM, et al. Even a moderate fluid removal rate during individualised haemodialysis session times is associated with decreased patient survival. Blood Purif. 2017; 44(2):89-97.

4 Sands JJ, Usvyat LA, Sullivan T, Segal JH, Zabetakis $\mathrm{P}$, Kotanko P, et al. Intradialytic hypotension: frequency, sources of variation and correlation with clinical outcome. Hemodial Int. 2014;18:415.

5 Zhou YL, Liu HL, Duan XF, Yao Y, Sun Y, Liu Q. Impact of sodium and ultrafiltration profiling on haemodialysis-related hypotension. Nephrol Dial Transplant. 2006 Nov;21(11): 3231-7.

6 Lopot F, Nejedlý B, Sulková S. Continuous blood volume monitoring and ultrafiltration control. Hemodial Int. 2000 Jan;4(1):8-14.
7 Onofriescu M, Mardare NG, Segall L, Voroneanu L, Cuşai C, Hogaş S, et al. Randomized trial of bioelectrical impedance analysis versus clinical criteria for guiding ultrafiltration in hemodialysis patients: effects on blood pressure, hydration status, and arterial stiffness. Int Urol Nephrol. 2012 Apr;44(2):583-91.

8 Sjolund J, Garcia Anton D, Bayes LY, Hoekstra T, Dekker FW, Munoz Mendoza J. Diuretics, limited ultrafiltration, and residual renal function in incident hemodialysis patients: a case series. Semin Dial. 2016 Sep;29(5):410-5.

9 Racette SB, Weiss EP, Schechtman KB, Steger-May K, Villareal DT, Obert KA, et al. Influence of weekend lifestyle patterns on body weight. Obesity. 2008 Aug;16(8):1826-30.

10 Saran R, Bragg-Gresham JL, Levin NW, Twardowski ZJ, Wizemann V, Saito A, et al. Longer treatment time and slower ultrafiltration in hemodialysis: associations with reduced mortality in the DOPPS. Kidney Int. 2006 Apr;69(7):1222-8.

11 Flythe JE, Kimmel SE, Brunelli SM. Rapid fluid removal during dialysis is associated with cardiovascular morbidity and mortality. Kidney Int. 2011;79:250-7.

12 Tugman MJ, Narendra JH, Li Q, Wang Y, Hinderliter AL, Brunelli SM, et al. Contemp ultrafiltration-profiled hemodialysis to reduce dialysis-related cardiovascular stress: study protocol for a randomized controlled trial. Clin Trials Commun. 2019 Jul 19;15:100415.

13 Murali KM, Mullan J, Roodenrys S, Hassan HC, Lambert K, Lonergan M. Strategies to improve dietary, fluid, dialysis or medication adherence in patients with end stage kidney disease on dialysis: a systematic review and meta-analysis of randomized intervention trials. PLoS One. 2019 Jan 29;14(1):e0211479.

14 Hur E, Usta M, Toz H, Asci G, Wabel P, Kahvecioglu $S$, et al. Effect of fluid management guided by bioimpedance spectroscopy on cardiovascular parameters in hemodialysis patients: a randomized controlled trial. Am J Kidney Dis. 2013;61(6):957-65.

15 Davies SJ, Caskey FJ, Coyle D, Lindley E, Macdonald J, Mitra S, et al. Rationale and design of BISTRO: a randomized controlled trial to determine whether bioimpedance spectroscopy-guided fluid management maintains residual kidney function in incident haemodialysis patients. BMC Nephrol. 2017; $18: 138$.

16 Cheuvront SN, Carter R 3rd, Montain SJ, Sawka MN. Daily body mass variability and stability in active men undergoing exerciseheat stress. Int J Sport Nutr Exerc Metab. 2004 Oct;14(5):532-40. 